\title{
ELECTROMYOGRAPHIC ACTIVITY OF STERNOCLEIDOMASTOID AND MASTICATORY MUSCLES IN PATIENTS WITH VESTIBULAR LESIONS
}

\author{
Gianluca M. TARTAGLIA ${ }^{1}$, Stefania BAROZZI ${ }^{2}$, Federico MARIN ${ }^{3}$, Antonio CESARANI ${ }^{4}$, Virgilio F. FERRARIO ${ }^{5}$
}

\begin{abstract}
1- DDS, PhD, Research Associate; Functional Anatomy Research Center (FARC), Laboratory of Functional Anatomy of the Stomatognathic Apparatus (LAFAS), Department of Human Morphology, School of Medicine and Surgery, University of Milano, Italy.

2- MD, PhD, Assistant Professor; Department of Otorhinolaryngology and Ophthalmology, School of Medicine and Surgery, University of Milano, Italy; Foundation Hospital Maggiore Policlinico, Mangiagalli and Regina Elena, Milano, Italy.

3- MD, PhD, Research Assistant; Functional Anatomy Research Center (FARC), Laboratory of Functional Anatomy of the Stomatognathic Apparatus (LAFAS), Department of Human Morphology, School of Medicine and Surgery, University of Milano, Italy.

4- MD, Professor; Department of Otorhinolaryngology and Ophthalmology, School of Medicine and Surgery, University of Milano, Italy; Foundation Hospital Maggiore Policlinico, Mangiagalli and Regina Elena, Milano, Italy.

5- MD, PhD, Professor; Functional Anatomy Research Center (FARC), Laboratory of Functional Anatomy of the Stomatognathic Apparatus (LAFAS), Department of Human Morphology, School of Medicine and Surgery, University of Milano, Italy.
\end{abstract}

Correspondence address: Prof. Virgilio F. Ferrario - Dipartimento di Morfologia Umana - via Mangiagalli 31 - I-20133 Milano - Italy. Phone: +39 - 02503 15407. Fax +39 - 0250315387 - e-mail farc@unimi.it

Received: March 20, 2008 - Modification: May 06, 2008 - Accepted: May 12, 2008

\begin{abstract}
$T$ his study evaluated the electromyographic characteristics of masticatory and neck muscles in subjects with vestibular lesions. Surface electromyography of the masseter, temporalis and sternocleidomastoid muscles was performed in 19 patients with Ménière's disease, 12 patients with an acute peripheral vestibular lesion, and 19 control subjects matched for sex and age. During maximum voluntary clenching, patients with peripheral vestibular lesions had the highest co-contraction of the sternocleidomastoid muscle (analysis of covariance, $\mathrm{p}=0.02$ ), the control subjects had the smallest values, and the patients with Ménière's disease had intermediate values. The control subjects had larger standardized muscle activities than the other patient groups ( $\mathrm{p}=0.001$ ). In conclusion, during maximum voluntary tooth clenching, patients with vestibular alterations have both more active neck muscles, and less active masticatory muscles than normal controls. Results underline the importance of a more inclusive craniocervical assessment of patients with vestibular lesions.
\end{abstract}

Key words: Masticatory muscles. Ménière’s disease. Peripheral vestibular lesion. Sternocleidomastoid

\section{INTRODUCTION}

The vestibular system permits the dynamic interaction of subjects with the environment in harmony with the force of gravity. In the brainstem, the elaboration of labyrinth, visual and somatosensory inputs generates reflex mechanisms directed to the oculomotor control and to the coordination of postural muscles. One of the main functions of the vestibular system is the stabilization of the head in the gravitational field and during movements. Anatomical and functional connections between the vestibular system and the neck muscles are well known ${ }^{18}$.

The sternocleidomastoid muscle (SCM) counteracts the extensor muscles of the neck to stabilize the head. Auditory inputs can modulate SCM activity: loud monaural clicks generate an initial inhibitory potential in the motoneurons controlling the tonically contracting ipsilateral SCM (vestibular evoked myogenic potentials). Vestibulospinal neurons of saccular origin mainly project to the motoneurons of the ipsilateral SCM and have inhibitory activity, whereas the same inputs have excitatory effect on neck extensor muscles $^{18,28}$.

The role played by the vestibular system on motor control mechanisms of masticatory muscles is less known. They are supposed to be involved not only in chewing and speech, but also in maintaining jaw posture away from its rest position in static and dynamic conditions ${ }^{20,21}$. Vestibular inputs can modulate the activity of trigeminal motor units innervating masticatory muscles as shown by both neurophysiological and anatomical studies ${ }^{11,17}$. Interactions between auditory and trigeminally innervated structures have also been demonstrated at various peripheral levels ${ }^{23,24}$.

Literature on the relationship between vestibular pathologies and cervical or jaw muscles is limited. A high 
prevalence of signs and symptoms of temporomandibular disorders (TMD), as well as of cranio-cervical disorders has been found in patients diagnosed with Ménière's disease ${ }^{3,5}$. Also, a dental origin for Ménière's disease has recently been proposed ${ }^{13}$.

Ménière's disease is a clinical disorder defined as the idiopathic syndrome of endolymphatic hydrops and characterized by the presence of recurrent spontaneous episodic vertigo, hearing loss, aural fullness and tinnitus. In most cases only one ear is involved, but both ears may be affected in about $15 \%$ of patients. The definitive spell of Ménière's disease is a spontaneous rotational vertigo lasting at least $20 \mathrm{~min}$ (commonly several hours), often prostrating and accompanied by nausea and commonly by vomiting or retching. Consciousness is not lost. During the definitive episode, horizontal rotary nystagmus is always present. The endolymphatic hydrops can only be demonstrated with certainty after death by the histopathologic study of the temporal bones ${ }^{26}$.

One of the disorders to be considered in the differential diagnosis of Ménière's disease is an acute peripheral vestibular lesion. This entity is a severe and prolonged vertigo provoked by a sudden impairment of a unilateral vestibular pathway including labyrinth, vestibular nerve or nuclei. A vestibular neuritis is often responsible for this lesion.

Our clinical experience, as well as previous investigations in patients with Ménière's disease $\mathrm{e}^{5}$ or peripheral vestibular lesion, report tenderness to palpation of the neck and masticatory (masseter and anterior temporalis) muscles. Also, symptom relief (mainly vertigo, but also tinnitus and feeling of fullness in the ear) has been described in these patients after treatment of TMD and cervical spine disorders ${ }^{4,27}$. Eidelman ${ }^{13}$ reported disappearance of symptoms of Ménière's disease after removal of intraosseous dental pathology. Ciancaglini, et al. ${ }^{8}$ found an increasing prevalence of ear symptoms in patients with TMD, with a direct relationship between deafness and severity of temporomandibular arthropathy ${ }^{8}$.

Along with these clinical assessments, no quantitative studies on the activity of cervical and head muscles, which have been reported to have some role in the development and maintenance of TMD and cranio-cervical disorders ${ }^{2,16}$, have been performed in patients with Ménière's disease or vestibular disturbances. Previous investigations analyzed the masticatory function in patients with TMD, and found surface electromyography (EMG) a useful diagnostic tool ${ }^{1,16,19,25}$. Indeed, TMD is a complex disease, and its nature has not yet been completely understood ${ }^{19}$. In some patients, altered occlusal conditions may be a factor in triggering abnormal muscular activity ${ }^{8}$, and actual occlusal alterations have been found in both longitudinal and cross-sectional investigations $\mathrm{s}^{7,12}$.

The aim of this study was to evaluate the EMG characteristics of the masseter, temporalis anterior and SCM muscles in two common disorders affecting the vestibular system, which are responsible for disabling vertigo: Ménière's disease and peripheral vestibular lesion. In particular, the influence of tooth contact (occlusion) on the standardized EMG activity will be investigated. If dental contact may provoke variations in EMG activity in patients with vestibular system disorders different from those found in normal controls, dental alterations may be responsible for the vestibular disorder, and the assessment of these patients should include an accurate dental evaluation ${ }^{3,5,13,27}$.

\section{MATERIAL AND METHODS}

\section{Patients}

Fifty adult subjects (23 men, 27 women, age range 3381 years) volunteered for the study after a detailed explanation of the experimental protocol. The subjects were either outpatients at the Ear, Nose and Throat (ENT) Department of Milan University medical school, or patients referred from a private dental office. The study protocol was approved by the local research ethics committee.

The ENT patients were either suffering from unilateral Ménière's disease (19 patients aged 35-70 years), or had had an acute peripheral vestibular lesion (12 patients aged 33-81 years). They all were examined during a symptomfree period, and recruited during a follow-up examination. The inclusion criteria comprised willingness to participate, no acute ENT disturbances, no dental problems or TMD, and no neck complains (in particular, no whiplash injury). The diagnosis of Ménière's disease was made according to the guidelines of the American Academy of OtolaryngologyHead and Neck Surgery ${ }^{9}$ : a history of at least two definitive spontaneous episodes of vertigo lasting $20 \mathrm{~min}$ or longer, tinnitus or aural fullness, and audiometrically documented sensorineural hearing loss of at least $60 \mathrm{~dB}$ on at least one occasion, while other pathology was excluded. Peripheral vestibular lesions were defined by canal paresis, calculated using Jongkees' formula, superior to $25 \%$ at the bithermal caloric test according to the Hallpike technique.

The patients referred from a private dental office (19 subjects aged 47-66 years) were matched for sex and age with the ENT patients, and were recruited during routine dental control examinations. Medical examination and history collection allowed to include only subjects who did not suffer from dental problems or TMD, or had ENT disturbances (in particular, vestibular and acoustic complains), or neck problems (mainly whiplash injury). The "dental" patients were used as a control group.

Most patients (both ENT and dental) had several dental prostheses on both natural teeth and dental implants. The type of occlusal support was not used as an inclusion/ exclusion criterion as far as the subject did not complain of problems with his/her teeth and supporting structures.

\section{Experimental Protocol}

Surface EMG of the masseter, temporalis anterior and SCM muscles was performed in all subjects. EMG data collection was made by investigators blinded to the subject group, and only numerical sex codes were used.

The experimental protocol comprised two 
standardization recordings and a maximum voluntary clenching (MVC). The same instrumentation and EMG tests detailed in previous studies were used ${ }^{15,16}$. In brief, the left and right masseter, temporalis anterior and SCM muscles were examined, with surface electrodes (Duo-Trode; MyoTronics Inc., Seattle, WA, USA) positioned on the muscular bellies parallel to muscular fibers. A disposable reference electrode was applied to the forehead. During all recordings, the patients sat with their head unsupported and were asked to maintain a natural erect position. They were asked to clench as hard as possible.

EMG activity was recorded using a computerized instrument (Freely, De Götzen srl; Legnano, Italy). The signals were averaged over $25 \mathrm{~ms}$, with muscle activity assessed as the root mean square (rms) of the amplitude (unit: $\mu \mathrm{V}$ ). EMG signals were recorded for further analysis.

\section{MVC standardization recordings}

These recordings provide reference EMG values for the subsequent normalization ${ }^{15,16}$. Two 10 -mm thick cotton rolls were positioned on the mandibular second premolar/first molars of each patient, and a 5-s MVC was recorded. For each of the four analyzed muscles, the mean EMG potential (rms of the amplitude) was set at $100 \%$, and all EMG potentials obtained during MVC directly performed on the occlusal surfaces (see below) were expressed as a percentage of this value (unit: $\mu \mathrm{V} / \mu \mathrm{V} \times 100$ ).

In addition, for standardization of SCM potentials, a maximal head-neck rotation without moving shoulders, blocked by one experimenter, was performed. The head was moved slowly on each side, and the subject remained in the extreme right and left positions for approximately $5 \mathrm{~s}^{15}$. During head-neck rotation, the contralateral SCM muscle is maximally activated. The mean EMG potentials obtained during head-neck rotation (rms of the amplitude) were set at $100 \%$. Standardized potentials were used to calculate the cervical load (see below).

\section{MVC in intercuspal position}

The patient was invited to clench as hard as possible with the teeth in the maximal intercuspal position, maintaining the same level of contraction for $5 \mathrm{~s}$. For each patient, the $3 \mathrm{~s}$ with the most constant rms EMG signal were automatically selected by the software, and the EMG potential were normalized as detailed before (EMG amplitude on occlusal surfaces divided by the mean EMG amplitude of the normalization record on the cotton rolls). The test was repeated three times, and the obtained values were averaged. Clenching did not provoke muscular/joint pain in either of the conditions (MVC on cotton rolls/ occlusal surfaces).

\section{EMG variables}

Some of the analyzed variables (standardized muscular activity; percentage overlapping coefficient, POC; Torque Coefficient, TC) had already been found to well discriminate among different groups of TMD patients, patients with neck disorders, and control subjects ${ }^{15,25}$. In addition, the relative muscular activities of the masseter and temporalis muscles would indicate the position of the occlusal center of gravity: more anterior positions increase the articular load ${ }^{14}$. SCM muscle symmetry and co-contraction during MVC were assessed considering the relationships between the vestibular system and the neck ${ }^{18,28}$.

The mean (left and right masseter and temporalis) total muscle activities were computed as the areas of the standardized EMG potentials (normalized rms amplitude) over time (unit: $\mu \mathrm{V} / \mu \mathrm{V} \cdot \mathrm{s} \%$ ). The EMG waves of paired muscles (symmetry; masseter, temporalis, and SCM) were compared by computing the POC index (unit: \%) ) $^{15,16}$.

Because an unbalanced contractile activity of contralateral masseter and temporalis muscles, for example right temporalis and left masseter, might give rise to a potential lateral displacing component, TC (unit \%) was calculated by superimposing the right temporalis plus left masseter normalized EMG amplitudes over the left temporalis plus right masseter normalized EMG amplitudes ${ }^{15,16}$ : the area of superimposition was assessed as a percentage of the total EMG amplitudes.

The standardized muscular activities of the masseter and temporalis muscles were compared by computing an anteroposterior coefficient (APC, unit \%) as the ratio between the non-overlapped and the overlapped masseter and temporalis muscle areas of both sides ${ }^{15}$. When standardized muscular potentials are not balanced between the two analyzed masticatory muscles, the occlusal center of gravity (MVC on the occlusal surfaces as compared to MVC on the cotton rolls) might be displaced onwards (temporalis prevalent) or backwards (masseter prevalent).

For the SCM muscle, a "cervical load" (percentage of co-contraction, unit: $\%)^{15}$ was assessed as the percentage ratio between the SCM muscle potentials recorded during MVC (this should be a submaximal contraction for SCM muscle) and the muscle potentials obtained during the maximum contraction standardization task (contralateral neck rotation against resistance).

Reproducibility of surface EMG measurements was previously reported, showing a good accuracy of the measurements, without random errors ${ }^{15}$.

\section{Statistical Analysis}

Descriptive statistics were computed for all variables within patient group and sex. Data were compared using 2way factorial analyses of variance, and 2-way factorial analyses of covariance (factor 1: patient group [normal subjects, Ménière's disease patients, patients with peripheral vestibular lesions]; factor 2: sex; the interaction patient group vs. sex was also computed). Age was used as a covariate. Post-hoc tests were performed by unpaired Student's t tests, Bonferroni correction for multiple testing. Categorical variables were compared by chi-square tests.

The level of significance was set at $5 \%$ for all statistical analyses. For multiple comparisons, a p value of 0.003 was used $(0.05 / 15)$. 


\section{RESULTS}

The sex distribution of the 3 patient groups was not statistically significant (Table 1 , Chi-square test $=0.207,2$ degrees of freedom, $\mathrm{p}>0.05$ ). On average, patients with Ménière's disease were significantly younger than patients with peripheral vestibular lesions and control patients (analysis of variance, $\mathrm{p}<0.001$ for factor "group"; post-hoc Student's t, $\mathrm{p}=0.002$, Ménière's disease vs. peripheral vestibular lesion; $\mathrm{p}<0.001$, Ménière's disease vs. control group).

Considering the age differences, EMG indices were compared by analysis of covariance (age as covariate). For all three indices of muscular symmetry, no significant group

TABLE 1- Demographic data and EMG indices in 50 adult subjects (mean and standard deviation)

\begin{tabular}{|c|c|c|c|c|c|c|c|c|c|c|}
\hline & \multirow[b]{2}{*}{ Unit } & \multicolumn{2}{|c|}{ Ménière's disease } & \multicolumn{2}{|c|}{ Peripheral vestibular lesion } & \multicolumn{2}{|c|}{ Control } & \multicolumn{3}{|c|}{ Comparison } \\
\hline & & Men & Women & Men & Women & Men & Women & Group & Sex & Group vs. S \\
\hline $\mathrm{N}$ & & 8 & 11 & 6 & 6 & 9 & 10 & & & \\
\hline Age & Y & $55.3(10.2)$ & $43.8(5.4)$ & $61.3(9.9)$ & $65.7(17.3)$ & $57.2(6.7)$ & $54.7(6.6)$ & $<0.001$ & NS & NS \\
\hline POC T & $\%$ & $80.8(10.8)$ & $85.8(4.4)$ & $86.5(2.3)$ & $78.9(9.9)$ & $86.0(3.1)$ & $85.5(4.5)$ & NS & NS & NS \\
\hline POC M & $\%$ & $81.9(9.0)$ & $84.2(4.5)$ & $86.5(4.1)$ & $83.0(8.3)$ & $75.2(17.8)$ & 84.8 (4.9) & NS & NS & NS \\
\hline POC SCM & $\%$ & $81.3(3.9)$ & $81.7(7.2)$ & $85.1(3.6)$ & $81.6(11.1)$ & $82.9(5.4)$ & $79.2(6.2)$ & NS & NS & NS \\
\hline TC & $\%$ & $86.8(4.2)$ & $90.0(1.9)$ & $91.0(1.9)$ & $87.5(4.0)$ & $83.3(11.4)$ & $89.7(9.3)$ & NS & NS & NS \\
\hline APC & $\%$ & $79.3(13.0)$ & $84.6(8.4)$ & $86.9(6.6)$ & $85.0(7.3)$ & $78.0(12.6)$ & $80.7(9.3)$ & NS & NS & NS \\
\hline Cervical Load & $\%$ & $29.3(37.4)$ & $16.8(10.0)$ & $58.5(37.3)$ & $26.6(29.9)$ & $25.0(16.9)$ & $9.2(5.2)$ & 0.035 & 0.012 & NS \\
\hline $\begin{array}{l}\text { Activity } \\
\text { standardized }\end{array}$ & $\begin{array}{c}\mu \mathrm{V} / \mu \mathrm{V} \cdot \mathrm{s} \\
\%\end{array}$ & $122.5(49.9)$ & $81.5(14.0)$ & 99.7 (33.2) & $80.3(28.4)$ & $153.0(78.3)$ & $156.9(68.6)$ & 0.001 & NS & NS \\
\hline
\end{tabular}

POC: percentage overlapping coefficient (index of left-right muscular symmetry); T: temporalis anterior muscle; M: masseter muscle; SCM: sternocleidomastoid muscle; TC: torque coefficient (potential lateral displacing component); APC: anteroposterior coefficient (relative activities of masseter and temporalis muscles); Cervical load: contraction of SCM during MVC as \% of standardization potentials; Comparison: $p$ values from a 2-way factorial analysis of variance (for age) or covariance (for all other variables; age as covariate): factor 1: group (Ménière's disease, peripheral vestibular lesion, control); factor 2: sex; interaction dental group vs. sex) NS, non-significant ( $p>0.05)$.

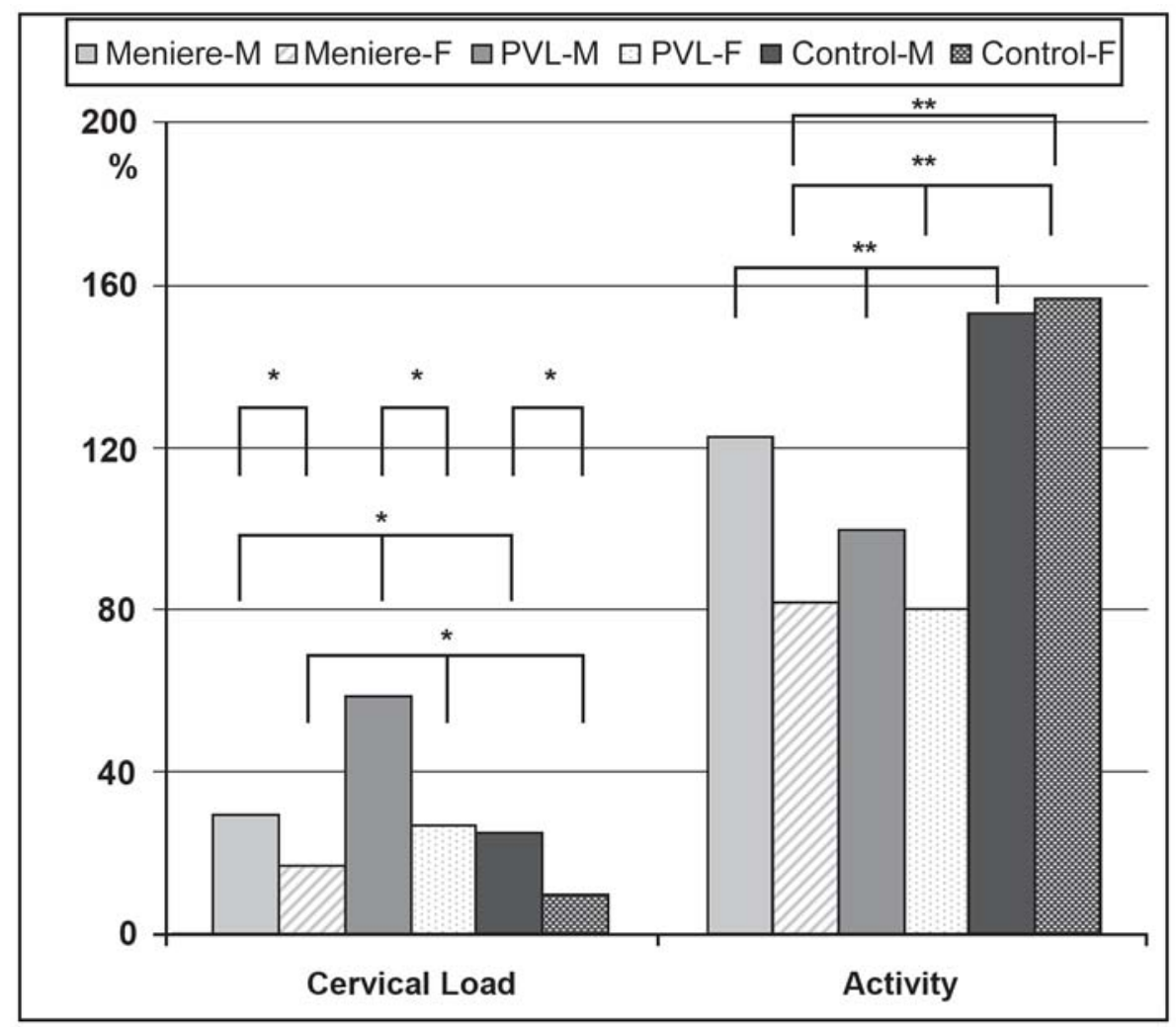

FIGURE 1- Standardized activity and cervical load (co-contraction of SCM during MVC) in male (M) and female (F) patients with Ménière's disease, peripheral vestibular lesion (PVL), and control patients (mean values). * $p<0.05 ;{ }^{* *} p<0.01$ (analysis of covariance, Student's t-test). 
or sex differences were found ( $>00.05)$. The torque and the anteroposterior coefficients did not differ among groups or between sexes.

The "cervical load" (percentage of co-contraction of the SCM muscle during MVC) was significantly larger in men than in women $(\mathrm{p}=0.012)$. The patients with peripheral vestibular lesions had the highest co-contraction of SCM muscle during teeth clenching $(\mathrm{p}=0.035)$, control patients had the smallest values, and patients with Ménière's disease had intermediate values (no significant post-hoc tests, $\mathrm{p}>0.003$ in all occasions) (Figure 1).

Significant among-group differences were found also for the standardized muscle activities $(\mathrm{p}=0.001)$ : on average, the control subjects had larger values than the other two patient groups, with a significant post-hoc comparison (control women vs. women with Ménière's disease, $\mathrm{p}=0.003)$.

\section{DISCUSSION}

Ménière's disease is a disabling disorder where recurrent aural vertigo is accompanied by tinnitus and deafness. This disease was first described by Prosper Ménière in 1861 and, in 1938, it was associated with endolimphatic hydrops. People in their fifties are those most often affected, but younger and older persons can also suffer from the disease ${ }^{13}$. In the last century, the estimated incidence in Italy has been reported to be 8.2 per $100,000^{6}$, in line with more recent reports worldwide ${ }^{4,22}$.

Treatment is most often symptomatic, involving dietary, pharmacological and surgical options, as well as acupuncture, physical therapy, and psychological support ${ }^{4,26}$. The variety of treatments demonstrates the lack of full knowledge of a disease whose definite diagnosis can be demonstrated only by histopathologic analysis of the endolymphatic sac.

Patients with Ménière's disease have been reported to suffer also from symptoms of TMD and cranio-cervicomandibular disorders, and to be positive to several signs typical of TMD and neck alterations ${ }^{3,5,13}$. A combined assessment of vestibular and stomatognathic function (and malfunctioning) emerges in the global evaluation of patients with head and face symptoms ${ }^{1,8,13}$. The treatment of TMD and cervical spine disorders seems to be effective also for distinctive vestibular and auditory symptom relief ${ }^{4,27}$, and this new therapeutic option for Ménière's disease has recently been proposed ${ }^{4}$.

The relationship between inner ear, jaw and neck disturbances has several anatomical bases: connections between neck and masticatory muscles, and peripheral and central vestibular and acoustic pathways, have been demonstrated in humans and mammals ${ }^{2,11,17,18,23,24,28}$.

In contrast, the literature does not report quantitative assessments on the activity of cervical and jaw muscles in patients with Ménière's disease or vestibular disturbances; these muscles can be assessed non-invasively by surface $\mathrm{EMG}^{16}$. Considering the limitations of surface EMG, a correct assessment should be performed with standardized (normalized) potentials, thus removing most of biological and technical noise ${ }^{10}$. In particular, the current standardization allows to determine the role of tooth contact (dental occlusion) in muscle activity ${ }^{15}$.

Peripheral vestibular lesions are among the disorders that could mimic Ménière's disease. Patients with a diagnosis of unilateral vestibular hypofunction other than Ménière's disease were used in the current study to verify whether the relationships (if any) between neck and jaw muscle activity and inner ear disorders were typical of Ménière's disease or they were more generally involved in vestibular disorders.

In the two groups of patients analyzed in the present study, the mean standardized masticatory muscular activity during the MVC test was significantly different from that found in control patients: on average, both the patients with Ménière's disease and those with peripheral vestibular lesions had a reduced activity. In addition, patients with peripheral vestibular lesions had significantly larger cocontractions of the SCM muscle during MVC ("cervical load") than control subjects.

The reduced standardized masticatory muscle activity may originate from a functionally unstable dental occlusion $^{19}$. Clenching on the cotton rolls reduced the proprioceptive inputs from this unstable occlusion, and allowed a more efficient contraction of masticatory muscles. In the patients with peripheral vestibular lesions, the increased co-contraction of SCM muscle during tooth clenching (expressed as a percentage of its maximal contraction during contralateral head and neck rotation) may be required to control head position during feeding and deglutition during the phase of vestibular compensation. Following an acute unilateral vestibular hypofunction, a functional recovery of balance is allowed by the vestibular compensation that consists of the disappearance of all asymmetries in the ocular and spinal vestibular responses. An important phase of this mechanism is the sensory substitution supported by an increase of cervical and proprioceptive information to the vestibular nuclei. SCM activity counteracts the increased paravertebral posterior cervical muscles permitting head stabilization.

The group of patients affected by Ménière's disease had "cervical load" values intermediate between control subjects and patients with peripheral vestibular lesions. The fluctuating course of Ménière's disease that cannot assure a stable compensation of the vestibular impairment can be a factor for this difference.

Based on the results of the present investigations, alterations in the activity of the masseter, temporalis anterior and SCM muscles during standardized clenching were not characteristic of Ménière's disease, but they were found also in patients suffering from non-specific vestibular disturbances. It must be mentioned that the analyzed individuals represent a convenience sample, and the extrapolation of the present results to a wider population should be done with caution. 


\section{CONCLUSION}

In patients with vestibular alterations, either specific such as Ménière's disease, or non-specific like a peripheral vestibular lesion, tooth contact during MVC may provoke alterations in the standardized contractile activity of their jaw and neck muscles, different from those found in healthy controls. These findings, together with literature reports of symptom relief in patients with Ménière's disease after treatment of TMD, cervical spine disorders, and dental pathologies ${ }^{4,13}$, underline the importance of a more inclusive assessment of ENT and dental patients ${ }^{8,13}$. Apart from clinical examination, objective functional assessments should be performed, and standardized surface EMG of the masticatory and neck muscles could be made with minimal discomfort to the patient ${ }^{1}$.

\section{REFERENCES}

1- Angeles-Medina F, Nuno-Licona A, Alfaro-Moctezuma P, OsornoEscareno C. Development and application of reflexodent in the quantitative functional evaluation of chewing control in patients with temporomandibular joint dysfunction and a control group. Arch Med Res. 2000;31:197-201.

2- Bevilaqua-Grossi D, Chaves TC, Oliveira AS. Cervical spine signs and symptoms: perpetuating rather than predisposing factors for temporomandibular disorders in women. J Appl Oral Sci. 2007;15:25964.

3- Bjorne A, Agerberg G. Craniomandibular disorders in patients with Meniere's disease: a controlled study. J Orofac Pain. 1996;10:28-37.

4- Bjorne A, Agerberg G. Symptom relief after treatment of temporomandibular and cervical spine disorders in patients with Meniere's disease: a three-year follow-up. Cranio. 2003;21:50-60.

5- Bjorne A, Berven A, Agerberg G. Cervical signs and symptoms in patients with Ménière's disease: a controlled study. Cranio. 1998;16:194202.

6- Celestino D, Ralli G. Incidence of Meniere's disease in Italy. Am J Otol. 1991:12:135-58.

7- Ciancaglini R, Gherlone EF, Radaelli G. Unilateral temporomandibular disorder and asymmetry of occlusal contacts. J Prosthet Dent. 2003;89:1805 .

8- Ciancaglini R, Loreti P, Radaelli G. Ear, nose, and throat symptoms in patients with TMD: the association of symptoms according to severity of arthropathy. J Orofac Pain. 1994;8:293-7.

9- Committee on Hearing and Equilibrium guidelines for the diagnosis and evaluation of therapy in Ménière's disease. Otolaryngol Head Neck Surg. 1995;113:181-5

10- De Luca CJ. The use of surface electromyography in biomechanics. J Appl Biomech. 1997;13:135-63.

11- Deriu F, Tolu E, Rothwell JC. A short latency vestibulomasseteric reflex evoked by electrical stimulation over the mastoid in healthy humans J Physiol. 2003;553:267-79.

12- Egermark I, Magnusson T, Carlsson, GE. A 20-year follow-up of signs and symptoms of temporomandibular disorders and malocclusions in subjects with and without orthodontic treatment in childhood. Angle Orthod. 2003;73:109-15.
13- Eidelman D. Meniere's disease may be caused by common intraosseous dental pathology: diagnosis using the comparative compression sign. Med Hypotheses. 2007;68:389-92.

14- Ferrario VF, Sforza C, Tartaglia GM, Dellavia C. Immediate effect of a stabilization splint on masticatory muscle activity in temporomandibular disorder patients. J Oral Rehabil. 2002;29:810-5.

15- Ferrario VF, Tartaglia GM, Galletta A, Grassi GP, Sforza C. The influence of occlusion on jaw and neck muscle activity: a surface EMG study in healthy young adults. J Oral Rehabil. 2006;33:341-8.

16- Ferrario VF, Tartaglia GM, Luraghi FE, Sforza C. The use of surface electromyography as a tool in differentiating temporomandibular disorders from neck disorders. Man Ther. 2007;12:372-9.

17- Giaconi E, Deriu F, Tolu E, Cuccurazzu B, Yates BJ, Billing I. Transneuronal tracing of vestibulo-trigemnal pathways innervating the masseter muscle in the rat. Exp Brain Res. 2005;24:1-10.

18- Kushiro K, Zakir M, Ogawa Y, Sato H, Uchino Y. Saccular and utricular inputs to sternocleidomastoid motoneurons of decerebrate cats. Exp Brain Res. 1999;126:410-6.

19- Landulpho AB, Silva WAB, Silva FA, Vitti M. Electromyographic evaluation of masseter and anterior temporalis muscles in patients with temporomandibular disorders following interocclusal appliance treatment. J Oral Rehabil. 2004;31:95-8.

20- Lund JP, Olsson KA. The importance of reflexes and their control during jaw movement. Trends Neurosci. 1983;11:458-63.

21- Miralles R, Manns A, Nass X, Pasini C, Rocabado M. Influence of protrusive functions on electromyographic activity of elevator muscles. Cranio. 1987;5:324-32

22- Shojaku H, Watanabe Y, Fujisaka M, Tsubota M, Kobayashi K, Yasumura S, et al. Epidemiologic characteristics of definite Meniere's disease in Japan. A long-term survey of Toyama and Niigata prefectures. ORL J Otorhinolaryngol Relat Spec. 2005;67:305-9.

23- Shore SE, Vass Z, Wys NL, Altschuler RA. Trigeminal ganglion innervates the auditory brainstem. J Comp Neurol. 2000;419:271-85.

24- Shore SE, Zhou J. Somatosensory influence on the cochlear nucleus and beyond. Hear Res. 2006;216-217:90-9.

25- Tartaglia GM, Moreira Rodrigues da Silva MA, Bottini S, Sforza C, Ferrario VF. Masticatory muscle activity during maximum voluntary clench in different research diagnostic criteria for temporomandibular disorders (RDC/TMD) groups. Man Ther. 2008;13(5):434-40.

26- Thorp MA, James AL. Prosper Meniere. Lancet. 2005;366:2137-9.

27- Wright EF, Bifano SL. Tinnitus improvement through TMD therapy. J Am Dent Assoc. 1997;128:1424-32.

28- Wu CH, Young YH, Murofushi T. Tone burst-evoked myogenic potentials in human neck flexor and extensor. Acta Otolaryngol. 1999;119:741-4. 